\title{
Expression of p16 protein in infantile hemangioma
}

\author{
SHI WANG ${ }^{1 *}$ and WEI $\mathrm{ZHENG}^{2 *}$ \\ ${ }^{1}$ Department of Stomatology, The Third Central Hospital, Tianjin 300170; ${ }^{2}$ Department of Orthodontics, \\ Tianjin Stomatological Hospital, Nankai University, Tianjin 300041, P.R. China
}

Received September 17, 2014; Accepted June 5, 2015

DOI: $10.3892 / \mathrm{ol} .2015 .3418$

\begin{abstract}
The present study aimed to investigate the expression and significance of p16 in the occurrence, development and regression of infantile hemangioma (IH). The expression of p16 was examined in proliferating, involuting hemangioma and normal tissues using immunohistochemical techniques. The expression of p16 was significantly lower in proliferating hemangioma than in involuting hemangioma, and was significantly lower in the involuting hemangioma than in normal tissues. Significant differences were found between the three groups $(\mathrm{P}<0.05)$. The results indicate that $\mathrm{p} 16$ may be important in the regression of $\mathrm{IH}$ endothelial cells and in anti-angiogenesis. There is a certain association between p16 expression and the regression of hemangioma. This provides a theoretical basis for the further study of the pathological mechanisms of p16 in hemangioma and potential gene therapies that may treat this disease.
\end{abstract}

\section{Introduction}

Infantile hemangioma ( $\mathrm{IH}$ ) is the most common soft-tissue tumor of infancy. It is benign tumor with an incidence of up to $10 \%$. IH is typically not present at birth, however, it may arise following rapid proliferation within the first weeks of life. This is followed by a plateau stage, and a subsequent slow involution of the lesion, such that $60 \%$ of 4 -year-olds and $76 \%$ of 7-year-olds with the condition will experience complete regression of the hemangioma. Characterized by a proliferation of endothelial cells, the pathogenesis of hemangioma is currently unclear $(1,2)$. In recent years, the development of

Correspondence to: Mr. Shi Wang, Department of Stomatology, The Third Central Hospital, 83 Jintang Road, Tianjin 300170, P.R. China

E-mail: winer_v@126.com

Dr Wei Zheng, Department of Orthodontics, Tianjin Stomatological Hospital, Nankai University, 75 Dagubei Road, Tianjin 300041, P.R. China

E-mail: zhengweivictory@163.com

${ }^{*}$ Contributed equally

Key words: p16, hemangioma, immunohistochemistry advanced molecular and cellular biology technologies has led to more in depth research on the cell cycle and its regulation mechanisms. Tumor suppressor genes, with their unique structures, biological activity and essential roles in the cell cycle, have accordingly become an important area of investigation.

Tumor suppressor genes, also known as anti-oncogenes, are present in normal cells and inhibit cell transformation and tumor occurrence $(3,4)$. p16 is a tumor suppressor gene that is able to block the $G_{1}$ to $S$ phase transition of the cell cycle, thereby inhibiting cell proliferation; in certain tumors, abnormal expression of this gene and protein may also promote the apoptosis of tumor cells. Aberrant p16 underexpression may cause cell proliferation and lead to cancer, the mechanism of which has been confirmed through research conducted in numerous tumor types; p16 inactivation most commonly occurs as a result of gene mutation or $5^{\prime} \mathrm{CpG}$ island methylation (5). The p16 gene and its protein have been found to be altered in the majority of human primary tumors, including melanoma, head and neck cancers, glioma and gastric cancer $(6,7)$. However, the expression of p16 protein in IH has not been reported.

The purpose of the present study was to detect 16 protein expression by immunohistochemical methods in hemangioma and normal tissues, and to investigate the mechanism and significance of the protein in the occurrence, development and regression of hemangioma, in order to provide novel insights into the clinical treatment of hemangioma.

\section{Materials and methods}

Patients. Between January 2001 and June 2006, a total of 80 patients with $\mathrm{IH}$, who had undergone surgery and were pathologically diagnosed with hemangioma at Stomatological Hospital of Jiamusi University (Jiamusi, China), were enrolled in this study. Paraffin-embedded hemangioma tissue blocks and clinical data were obtained for 38 males and 42 females, who were of a minimum age of 2 months and a maximum age of 4 years. The hemangiomas were located on the scalp, forehead, eyelids, neck, back, arms, legs, hands or feet. The patients underwent no auxiliary treatment. Paraffin blocks were cut into $4-\mu \mathrm{m}$ thick sections, which were each cut into two pieces, for the detection of p16 protein and hematoxylin and eosin (HE) staining. After HE staining, the paraffin blocks were grouped, according to the Mulliken classification standard (8), into 40 cases of proliferating hemangioma and 40 cases of involuting hemangioma, with tumor-adjacent 
Table I. p16 expression in normal tissue, involuting hemangioma tissue and proliferating hemangioma tissue.

\begin{tabular}{lccccccr}
\hline & & \multicolumn{5}{c}{$\mathrm{p} 16$ expression, $\mathrm{n}$} \\
\cline { 3 - 6 } Groups & $\mathrm{n}$ & - & + & ++ & +++ & Positive rate, \% & P-value \\
\hline Normal tissue & 26 & 4 & 2 & 4 & 16 & 84.62 & $0.001^{\text {a }}$ \\
Involuting hemangioma & 40 & 19 & 4 & 7 & 10 & 52.50 & $0.018^{\mathrm{b}}$ \\
Proliferating hemangioma & 40 & 31 & 5 & 2 & 2 & 22.50 & $0.016^{\mathrm{c}}$ \\
\hline
\end{tabular}

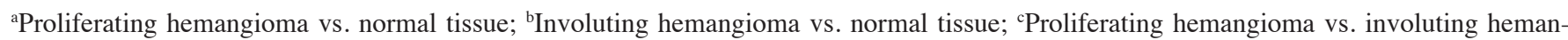
gioma.
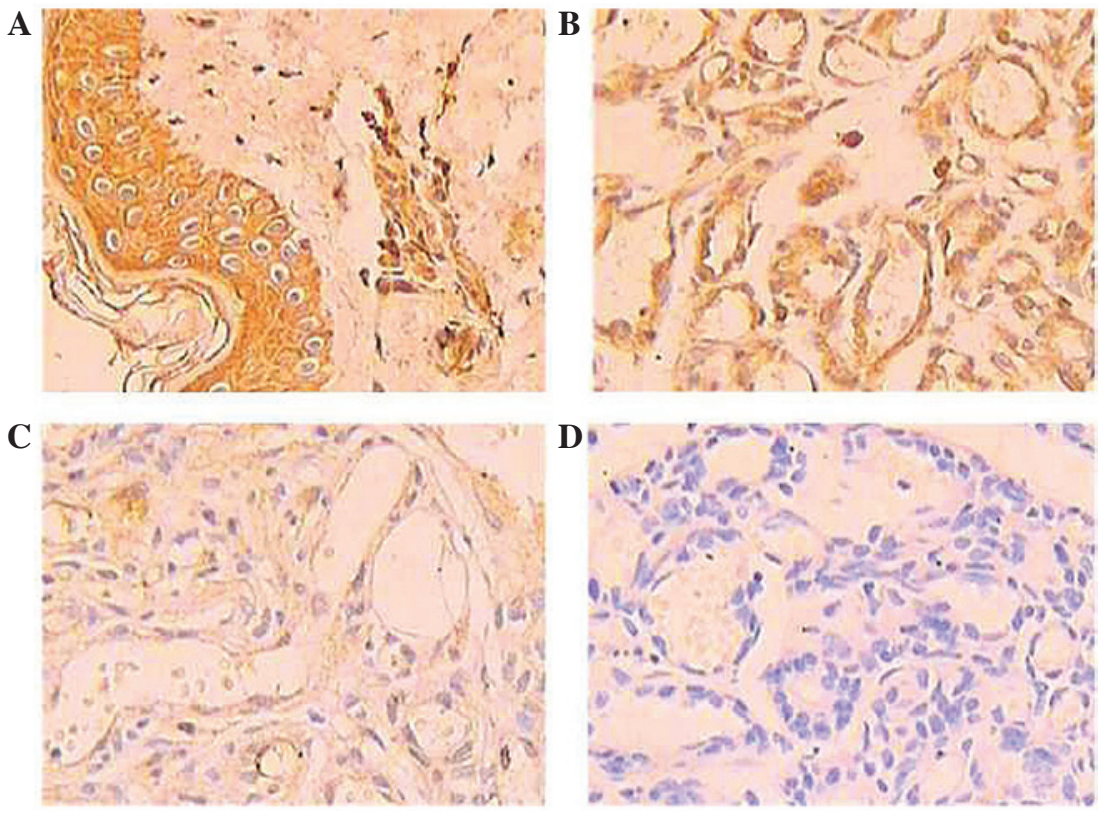

Figure 1. Immunohistochemical analysis. (A) Very strong expression of p16 protein in the normal tissue cells. (B) Strong expression of p16 protein in the involuting hemangioma. (C) Weak expression of p16 protein in the proliferating hemangioma. (D) Negative control where the primary p16 was replaced by phosphate-buffered saline. Magnification, x200.

normal tissues in 26 cases used as controls. The study was approved by the Ethics Committee of the Stomatological Hospital of Jiamusi University. Written informed consent was obtained from the patient's families.

Immunohistochemistry. The 4- $\mu \mathrm{m}$ sections were deparaffinized in xylene followed by hydration in a graded series of alcohols. Antigen retrieval was performed by immersing the section in $0.5 \mathrm{M}$ citrate buffer $(\mathrm{pH} 6)$ at room temperature, and placing it into a microwave oven for $20 \mathrm{~min}$. Endogenous peroxidase activity was blocked by incubation in $3 \%$ $\mathrm{H}_{2} \mathrm{O}_{2}$ for 20 min at room temperature. Subsequent to being rinsed in Tris-buffered saline (TBS; $\mathrm{pH}$ 7.4), the sections were incubated with primary rabbit anti-p16INK4a monoclonal antibody (Wuhan Boster Biological Technology Ltd., Wuhan, China; dilution, 1:2 in TBS) at $4^{\circ} \mathrm{C}$ overnight. In the negative control, the primary antibody was replaced with phosphate-buffered saline. Subsequent to being washed with TBS three times for $5 \mathrm{~min}$ each, the sections were incubated with a biotin-labeled goat anti-rabbit $\operatorname{IgG}$ secondary antibody (Wuhan Boster Biological Technology Ltd.). The sections were counterstained with 3-3'-diaminobenzidine (Wuhan Boster Biological Technology Ltd.) followed by hematoxylin. The slides were washed under running water and mounted with DPX. The protein expression was then scored as follows: negative $(-),<25 \%$ positive cells; weak $(+), 25-50 \%$ positive cells; strong $(++),>50-75 \%$ positive cells; and very strong $(+++),>75 \%$ positive cells.

Statistical analysis. The data are expressed as number of cases and percentage. Categorical data was analyzed using the $\chi^{2}$ test. SPSS 15.0 software (SPSS Inc., Chicago, IL, USA) was used for all data analyses. $\mathrm{P}<0.05$ was considered to indicate a statistically significant difference.

\section{Results}

Upon immunohistochemical staining, the positive expression of p16 protein was shown as brown-yellow granules, mainly in the cytoplasm and partially in the nucleus. The normal tissue cells exhibited a large number of brown-yellow granules, indicating that the expression of p16 protein was very strong 
(Fig. 1A), with a positive rate of $84.62 \%$. The expression level of p16 protein was different in the different types of hemangioma. In the involuting hemangioma, numerous brown granules were deposited in the endothelial cell, indicating that the expression of p16 protein was strong (Fig. 1B), with a positive rate of $52.5 \%$. In the proliferating hemangioma, less brown granules were deposited in the endothelial cells, indicating that the expression of p16 protein was weak (Fig. 1C), with a positive rate of $22.5 \%$. The expression level of p16 protein in the proliferating hemangioma was lower than that in the involuting hemangioma. The expression level of p16 protein in the involuting hemangioma was lower than that in normal tissue. These differences were statistically significant $(\mathrm{P}<0.05)$ (Table I).

\section{Discussion}

Molecular studies have shown that tumor development is closely associated with the cell cycle. Normal cells possess a dynamic balance between proliferation and the inhibition of proliferation, so as to ensure the normal growth of the organism. When this balance is broken, the components for promoting proliferation cause overexpression or the components for inhibiting proliferation cause underexpression; with this, cell proliferation becomes uncontrolled and tumors form. p16 is a tumor suppressor gene, located in chromosomal region $9 \mathrm{p} 21$, with a total length of $8.5 \mathrm{~kb}$ and containing 3 exons and 2 introns. The p16 protein is encoded by a single polypeptide chain of $15.84 \mathrm{kDa}$, containing 148 amino acid residues, and is involved in the regulation of cell growth. The p16 protein is a cyclin dependent kinase (CDK)4/CDK6 inhibitor, and CDK4/CDK6 is the key factor for the $G_{1} / S$ phase transition in the cell cycle. p16 protein is specifically combined with CDK4/CDK6, and its inactivation prevents the phosphorylation of $\mathrm{Rb}$ protein, which means that the $\mathrm{Rb}$ gene cannot relieve the inhibition of transcription factors, thereby preventing the cells from passing from the $G_{1}$ phase to the $\mathrm{S}$ phase. This therefore inhibits cell proliferation, making p16 a key negative regulatory factor in the process of cell proliferation (9-11). The p16 gene and its protein are altered in the majority of human primary tumors, including gliomas, melanoma, head and neck cancer and gastric cancer (12-14).

Inactivation (deletion or mutation) and 5' $\mathrm{CpG}$ island methylation are the main forms of change for the p16 protein. Kamb et al (15) analyzed 290 tumor cell lines and found that 133 of these cell lines, including lung cancer and leukemia cell lines, showed deletion of the p16 gene; the gene deletion rate was $25 \%$ in glioma and the mutation rate was $85 \%$. In breast cancer, and cancer of the head and neck, the mutation rates were 60 and $30 \%$, respectively. p16 gene deletion, mutation or hypermethylation cause cells to undergo uncontrolled proliferation, and are thus some of the major factors in the occurrence and development of tumors $(7,15)$.

Hemangioma is a benign tumor with endothelial cell hyperplasia; the condition occurs in infants, with a pathogenesis that is unknown. The current view is that endothelial cell hyperplasia, the aggregation process and microvessel lumen formation are the core of vascular tumor growth. A previous study revealed that endothelial cells in the proliferative phase were in an immature state and had strong proliferative capability (16).

The expression of p16 protein in IH was detected by immunohistochemistry in the present study. The experimental results showed that the expression level of p16 protein in the proliferating hemangioma was lower than that in the involuting hemangioma, with a significant difference $(\mathrm{P}<0.05)$, and that the expression level of p16 protein in the involuting hemangioma was lower than that in the normal tissue, with a significant difference $(\mathrm{P}<0.05)$. Based on the results of the present study, it is hypothesized that the p16 protein is present at a low level of expression in proliferating hemangioma and is only partially expressed by endothelial cells; it therefore cannot inhibit the hemangioma endothelial cell CDK4/ CDK6 activity, and is unable to prevent the cell from entering the $G_{1}$ phase to $S$ phase transition. Thus, cell proliferation becomes uncontrolled, leading to the excessive proliferation of the endothelial cells, accelerating hemangioma proliferation. In the period of hemangioma regression, the expression of p16 protein is increased, thus inhibiting the hemangioma endothelial cell CDK4/CDK6 activity, leading to cell cycle arrest, the inhibition of the proliferation of the hemangioma endothelial cells, and promoting the involution of $\mathrm{IH}$.

Hemangioma is a type of angiogenic disease (17). The results of the present study indicate that endothelial cell proliferation and angiogenesis are not the result of a single factor; each process may be a result of the participation of multiple genes and factors. The p16 protein was the first protein molecule identified to have tumor suppressor functions (9). Due to the high frequency of deletion and mutation in numerous primary tumors, restoring normal function of the p16 gene in tumor cells is a potential method of inhibiting tumor cell proliferation. The p16 gene has the following features: cDNA molecules are small and easy to manipulate; the p16 protein is also able to specifically inhibit CDK4/ CDK6 so, as an antitumor drug, it has a specific target; in comparison to other genes the mutation rate is high, and mutations are observed in numerous primary tumors (18). Therefore, the p16 gene may become an important candidate gene for gene therapy. By increasing the p16 protein level of a patient, it may be possible to prevent the proliferation of hemangioma endothelial cells.

From the results of the present study, we speculated that the p16 protein plays a role in inhibiting the proliferation of hemangioma endothelial cells and in anti-angiogenesis. There is a certain association between p16 and the regression of hemangioma. This provides a theoretical basis for the further study of the pathological mechanisms of p16 in hemangioma and potential gene therapies that may treat this disease.

\section{References}

1. Sans V, Dumas de la Roque ED, Berge J, Grenier N, Boralevi F, Mazereeuw-Hautier J, Lipsker D, Dupuis E, Ezzedine K, Vergnes P, et al: Propanolol for severe infantile hemangiomas: Follow-up report. Pediatrics 124: e423-e431, 2009.

2. Chen TS, Eichenfield LF and Friedlander SF: Infantile hemangiomas: An update on pathogenesis and therapy. Pediatrics 131: 99-108, 2013.

3. Yang B, House MG, Guo M, Herman JG and Clark DP: Promoter methylation profiles of tumor suppressor genes in intrahepatic and extrahepatic cholangiocarcinoma. Mod Pathol 18: 412-420, 2005.

4. Ekholm SV and Reed SI: Regulation of G(1) cyclin-dependent kinases in the mammalian cell cycle. Curr Opin Cell Biol 12: 676-684, 2000

5. Li J, Poi MJ and Tsai MD: Regulatory mechanisms of tumor suppressor P16(INK4A) and their relevance to cancer. Biochemistry 50: 5566-5582, 2011. 
6. Bachmann IM, Straume O and Akslen LA: Altered expression of cell cycle regulators Cyclin D1, p14, p16, CDK4 and Rb in nodular melanomas. Int J Oncol 25: 1559-1565, 2004.

7. Witkiewicz AK, Knudsen KE, Dicker AP and Knudsen ES The meaning of p16(ink4a) expression in tumors: Functional significance, clinical associations and future developments. Cell Cycle 10: 2497-2503, 2011.

8. Mulliken JB and Glowacki J: Hemangiomas and vascular malformations in infants and children: A classification based on endothelial characteristics. Plast Reconstr Surg 69: 412-422, 1982.

9. Serrano M, Hannon GJ and Beach D: A new regulatory motif in cell-cycle control causing specific inhibition of cyclin D/CDK4 Nature 366: 704-707, 1993.

10. Li W, Sanki A, Karim RZ, Thompson JF, Soon Lee C, Zhuang L, McCarthy SW and Scolyer RA: The role of cell cycle regulatory proteins in the pathogenesis of melanoma. Pathology 38 287-301, 2006.

11. Khoo CM, Carrasco DR, Bosenberg MW, Paik JH and Depinho RA: Ink4a/Arf tumor suppressor does not modulate the degenerative conditions or tumor spectrum of the telomerase-deficient mouse. Proc Natl Acad Sci U S A 104: 3931-3936, 2007.

12. Horbinski C, Nikiforova MN, Hagenkord JM, Hamilton RL and Pollack IF: Interplay among BRAF, p16, p53, and MIB1 in pediatric low-grade gliomas. Neuro Oncol 14: 777-789, 2012.
13. de Andrade BA, León JE, Carlos R, Delgado-Azañero W, Mosqueda-Taylor A and de Almeida OP: Immunohistochemical expression of p16, p21, p27 and cyclin D1 in oral nevi and melanoma. Head Neck Pathol 6: 297-304, 2012.

14. Goto T, Mizukami H, Shirahata A, Yokomizo K, Kitamura YH, Sakuraba K, Saito M, Ishibashi K, Kigawa G, Nemoto H, et al: Methylation of the p16 gene is frequently detected in lymphatic-invasive gastric cancer. Anticancer Res 30: 2701-2703, 2010

15. Kamb A, Gruis NA, Weaver-Feldhaus J, Liu Q, Harshman K, Tavtigian SV, Stockert E, Day RS III, Johnson BE and Skolnick MH: A cell cycle regulator potentially involved in genesis of many tumor types. Science 264: 436-440, 1994.

16. Lo K, Mihm M and Fay A: Current theories on the pathogenesis of infantile hemangioma. Semin Ophthalmol 24: 172-177, 2009.

17. Phung TL, Hochman M and Mihm MC: Current knowledge of the pathogenesis of infantile hemangiomas. Arch Facial Plast Surg 7: 319-321, 2005.

18. Volgareva G, Zavalishina L, Andreeva Y,Frank G, Krutikova E, Golovina D, Bliev A, Spitkovsky D, Ermilova V and Kisseljov F: Protein p16 as a marker of dysplastic and neoplastic alterations in cervical epithelial cells. BMC Cancer 4: 58, 2004. 